\title{
Challenging Cases in Urothelial Cancer: Case 21
}

\author{
Mark S. Soloway* \\ Memorial Physician Group, Division of Urology, Memorial Healthcare System, Aventura, FL, USA
}

This is a 65-year-old current and longtime cigarette smoker who presented to me with an 8 month history of intermittent gross hematuria and a diagnosis of muscle invasive urothelial cancer of the bladder. He has type 2 diabetes mellitus. He had symptomatic coronary artery disease and coronary artery stents were placed in 2015 . He currently has a good performance status.

He was initially evaluated by another urologist who identified a papillary and sessile tumor on the right side of the bladder located above the right ureteral orifice. The tumor appeared to be arising from a bladder diverticulum.

A CT scan indicated a normal upper urinary tract with no ureteral dilatation (Fig. 1). The bladder outline indicated an obvious mass on the right side consistent with the description of the endoscopy performed by the initial urologic surgeon (Fig. 2). Another image indicated a thick walled bladder without a mass (Fig. 3). An image with contrast identified many diverticuli as well as the mass (Fig. 4). There were no enlarged lymph nodes. Chest imaging was normal.

The pathology from the initial resection indicated a papillary high grade urothelial cancer with both papillary and glandular components. There were foci of

\footnotetext{
${ }^{*}$ Correspondence to: Mark S. Soloway, MD, Memorial Physician Group, Division of Urology, Memorial Healthcare System, Aventura, FL, USA. E-mail: mssoloway@yahoo.com.
}

carcinoma in situ. There was invasion into the muscularis propria.

I saw the patient for consultation in March 2021. He was already counseled that a radical cystoprostatectomy preferably with neoadjuvant chemotherapy would be the optimal treatment. He was very reluctant to have his bladder removed. He was referred to me for an opinion and treatment. The scans and pathology were reviewed and the information including the histology was confirmed.

To better assess the extent and location of the cancer I proceeded with another cystoscopy and TUR BT. I identified the right ureteral orifice and obvious residual sessile and focally papillary tumor posterior and lateral to the trigone (Fig. 5-7). The CT scan showed a bladder diverticulum however the lumen was not evident because of the prior resection and tumor. The rest of the bladder was entirely normal with the exception of a multiple diverticuli. I did not perform mucosal biopsies of normal appearing urothelium since its appearance was entirely normal and it was apparent to me that this was a unifocal tumor. I resected some of the visible tumor but knowing that there was a diagnosis of muscle invasive cancer, and a "complete" resection would not be possible I did not attempt an extensive TUR BT.

I had several lengthy discussions with the patient and his family, and I raised the consideration of a partial cystectomy although I emphasized that the optimal treatment would be to remove the entire blad- 

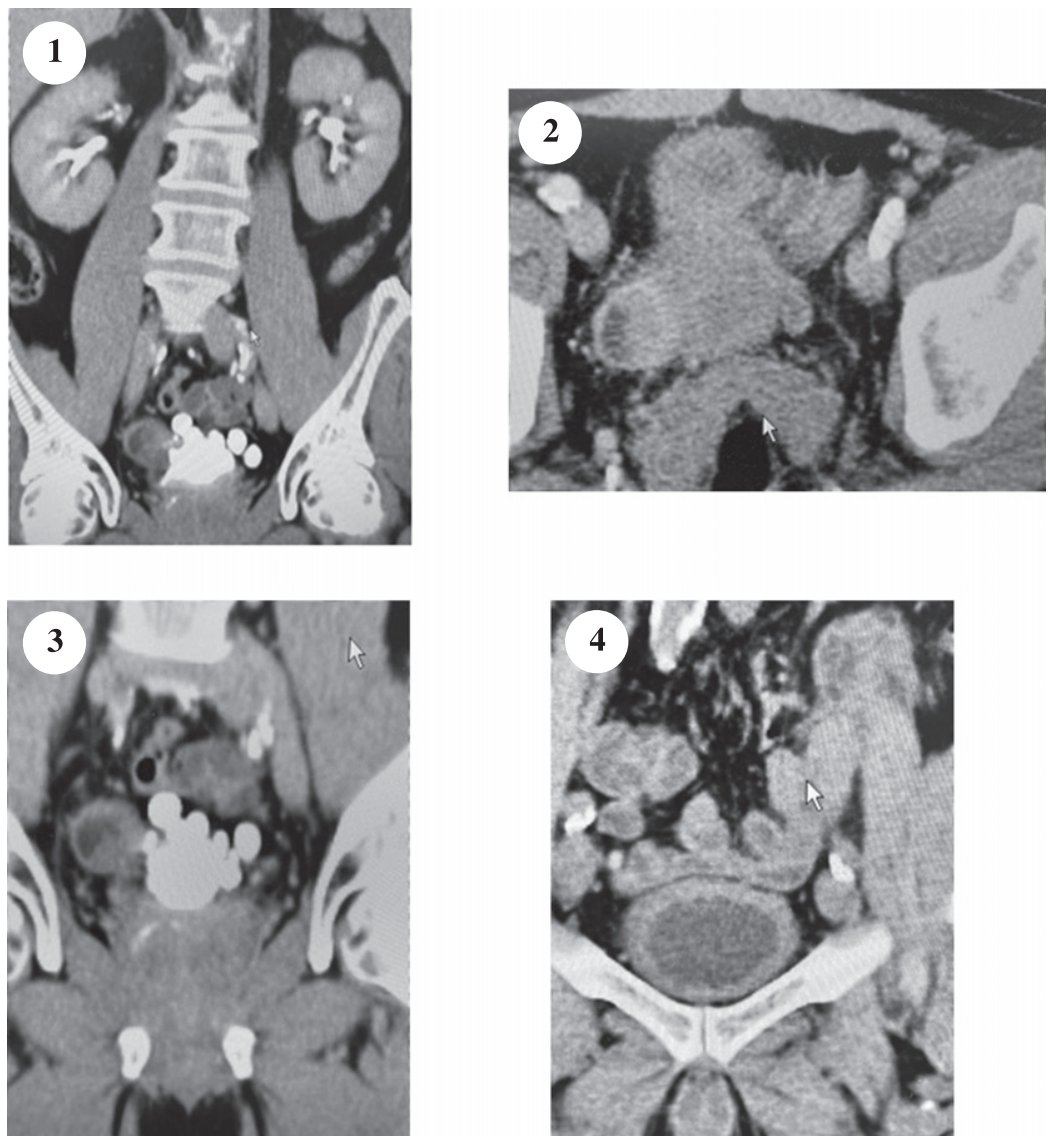

Fig. 1-4. The CT scan of the abdomen and pelvis indicate a normal upper urinary tract. There is a mass associated with a bladder diverticulum arising from the right side of the bladder. There are multiple bladder diverticuli as seen in the image post contrast. The bladder is moderately thick.
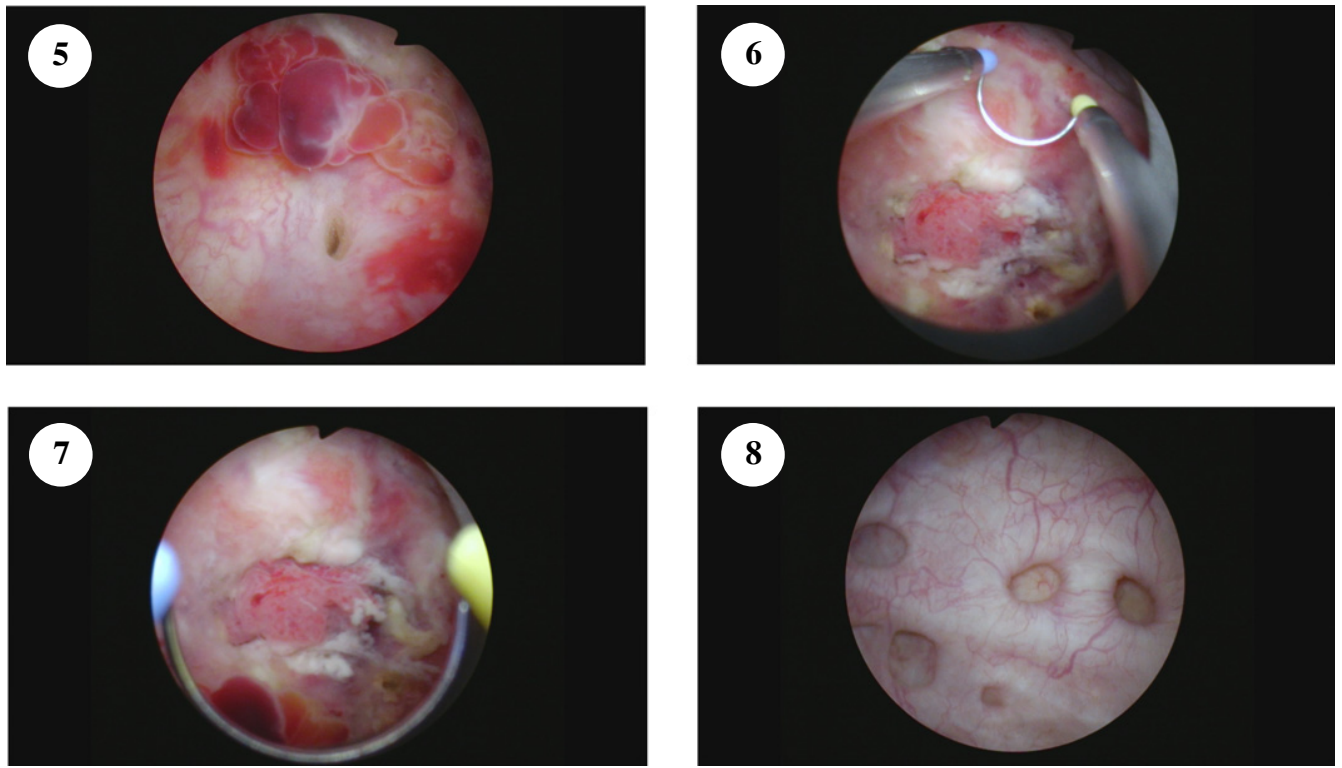

Fig. 5-8. The right ureteral orifice is identified in figure 5. The tumor is seen in figures 6-7. 
der preferably with neoadjuvant chemotherapy since the margin at the trigone would be close to the ureteral orifice and would likely require a reimplantation if a partial cystectomy was performed. I explained that the main concern was obtaining and adequate margin of normal bladder and thus avoid a local recurrence.

The intensive discussions about the approach and the patient's desire to avoid a total cystectomy was in large part a reason for a three month delay from my re TUR BT to his agreeing to proceed with surgery.

The patient agreed to have me attempt a partial cystectomy however, if frozen sections of the margins indicated urothelial cancer I could proceed with removal of the entire bladder and prostate and construct an orthotopic neobladder.

I proceeded with a standard midline lower abdominal incision with entry into the peritoneal cavity as per a standard radical cystectomy. The right lower ureter was mobilized. The vas deferens and obliterated obturator vessels were divided bilaterally to allow mobilization of the posterior and anterior bladder. A firm mass was easily palpated on the right side of the bladder (Fig. 9). The mass was about $3 \mathrm{~cm}$ in diameter. My impression was that a partial cystectomy might be feasible. The external iliac vessels were identified and palpated and consistent with the previous imaging there was no palpable lymph node enlargement.

Given the location of the tumor being lateral and posterior I did not think it was feasible to remove the tumor mass without opening the bladder.

I opened the bladder and separated the lateral portion of the bladder which consisted of the diverticulum and adjacent bladder tissue from the rest of the bladder (Fig. 10-11). The right ureter entered the lower edge of the tumor and was divided. The partial cystectomy was completed. The remaining bladder at the excision sites was palpably normal. I then provided a series of bladder tissue from the margins for frozen section. There was no tumor at the margins. The bladder was closed with a two layer closure. The right ureter was reimplanted using an extravesical end to side anastomosis with six -0 suture. A JJ stent was placed. A right extended pelvic lymph node dissection was performed. There was a firm small nodule of tissue at the base of the obliterated obturator vessel which was excised and submitted separately.

The final pathology indicated a high grade focally undifferentiated urothelial carcinoma with papillary and glandular areas. The tumor extended through the muscularis propria into the perivesical tissue. There
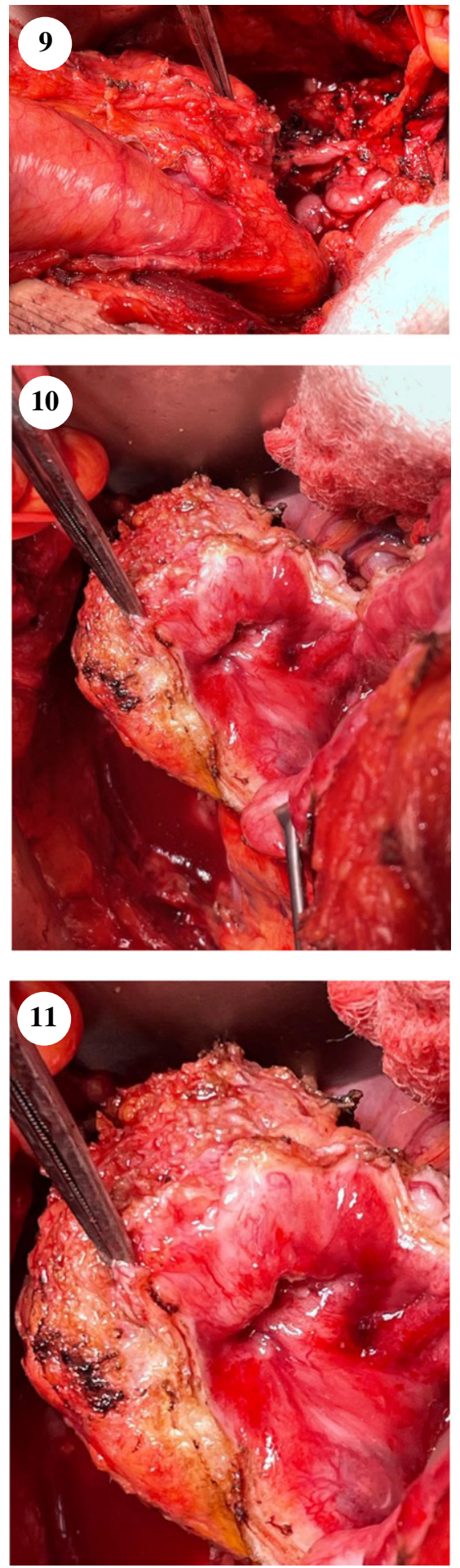

Fig. 9-11. The firm mass is seen in figure 9 with the instrument pointing to it. The mobilized right distal ureter is seen proximal to the bladder. Figures 10-11 show the tumor containing bladder diverticulum. 
was CIS. There was lymphovascular invasion. The pelvic lymph nodes did not have tumor however the small nodule had a $1 \mathrm{~mm}$ focus of metastatic urothelial cancer.

The patient has made an uneventful recovery. The question now remains as to whether he should be observed or receive systemic adjuvant therapy and, if so, whether this should be cisplatin based combination chemotherapy or a PD -1 or a PD-L1 immune check point inhibitor.

I wonder whether I should have insisted on a radical cystoprostatectomy. Would this change his prognosis? I have interpreted the literature on this topic to be that as long as one can attain an adequate surgical margin and this is the first tumor for the patient and the rest of the bladder is normal a partial cystectomy provides a similar chance of cure from the initial muscle invasive cancer than would a total cystectomy.

\section{INFORMED CONSENT}

Written informed consent for publication was obtained from the patient.

\section{ETHICAL CONSIDERATIONS}

The author has institutional review board approval for reviewing bladder cancer cases for outcome (MHS.2020.026).

\section{CONFLICT OF INTEREST}

Nothing to disclose. 